\title{
LA DIMENSION SPATIALE DES COLLABORATIONS POUR L'INNOVATION : UNE ANALYSE SUR DONNEES CIFRE (1981-2006)
}

\author{
SPATIAL PATTERNS OF PARTNERSHIPS DEDICATED TO \\ INNOVATION: A CIFRE DATA ANALYSIS FROM 1981 TO 2006
}

\author{
BOUBA-OLGA Olivier \\ Maître de conférences \\ Laboratoire CRIEF-TEIR EA2249 \\ Université de Poitiers \\ 93, avenue du recteur Pineau \\ 86022 Poitiers Cedex \\ obouba@univ-poitiers.fr \\ FERRU marie \\ Maître de conférences \\ Laboratoire CRIEF-TEIR EA2249 \\ Université de Poitiers \\ 93, avenue du recteur Pineau \\ 86022 Poitiers Cedex \\ marie.ferru@univ-poitiers.fr
}

Mots clés : Innovation, géographie, inertie, ressources, distance

Keywords : Innovation, geography, inertia, resources, distance

Classification JEL : O31, R12, O39

\footnotetext{
Nous remercions Liliane Bonnal, Dominique Pépin, Benoit Mulkay et les participants au colloque ASRDLF qui a eu lieu en 2009 à Clermont-Ferrand pour leurs remarques sur une première version de l'article et leurs conseils pour les analyses économétriques réalisées. Les erreurs éventuelles restent bien sûr de notre entière responsabilité.
} 
Résumé L'objectif de cet article est de se prononcer sur l'évolution de la dimension spatiale des collaborations pour l'innovation et sur l'existence d'éventuels effets de structure et notamment d'effets-taille. Nous développons dans un premier temps un modèle d'adoption des collaborateurs, qui met en évidence le rôle potentiel de la géographie des ressources et de l'histoire des relations passées (effets de lock-in) pour la dimension spatiale des collaborations. Nous nous appuyons dans un deuxième temps sur une base de données originale recensant plus de 14000 contrats CIFRE sur la période 1981-2006 et testons deux modèles économétriques complémentaires. Trois grands résultats ressortent de ce travail : la géographie des collaborations pour l'innovation est marquée par une forte inertie, l'existence d'effets régionaux et le rôle important d'effets-taille.

Summary The aim of this paper is to provide new insights about the dynamic of the geography of knowledge collaborations and structural effects, such as size effects. We propose, in a first part of the paper, a theoretical model of partners adoption that highlights the potential roles in the geography of collaborations of both, the geography of resources and the history of partnerships (lock in effects). Thanks to a database that collects 14000 Cifre contracts signed between 1981 and 2006, we realize, in a second part, two complementary econometric tests. Three important results are revealing: the geography of partnerships is structured by inertia, regional, size effects.

\section{$-1-$ \\ Introduction}

L'Université apparaît, d'un point de vue théorique et empirique, comme un partenaire essentiel pour l'innovation, en tant que source de nouvelles connaissances (GIBBONS et al. (1994) ; FELDMAN (1994); SAXENIAN (1994); ANSELIN et al. (1997)). Selon l'enquête CIS (community Innovation Survey) du SESSI (2006), un peu plus de $20 \%$ des établissements français collabore avec un laboratoire universitaire ou un organisme de recherche publique pour mener à bien leur projet d'innovation. Le poids de ces partenariats est par ailleurs croissant (GODIN et GINGRAS (1999); WAGNER et LEYDESDORFF (2005); HAGGEDOORN et ROIJAKKERS (2006)).

L'importance à la fois qualitative et quantitative de ces collaborations a conduit à de nombreuses investigations depuis une quinzaine d'années (GIBBONS et al. (1994) ; JOLY et MANGEMATIN (1996); ETZKOWITZ et al. (1998); CARAYOL (2003)). Certains cherchent à comprendre leur organisation, leur objet, etc. ; d'autres testent le lien entre différentes caractéristiques des partenaires (taille, secteurs d'activité, intensité technologique) et la propension à collaborer (pour un résumé de la littérature existante sur les collaborations science-industrie, voir Bergman (2010)). Compte tenu de la forte polarisation des activités d'innovation (LUNG (1997) ; PUGA (1999) ; LALLEMENT et al. (2007)), d'autres auteurs se sont focalisés sur la question essentielle de la dimension spatiale de ces collaborations. S'appuyant sur les modèles théoriques de la Nouvelle Economie Géographique, un ensemble d'auteurs (JAFFE (1989) ; Acs et al. (1991) ; FELDMAN (1994) ; FELDMAN et FLORIDA (1994) ; AUDRETSCH et FELDMAN (1996)) qui relève de la "géographie de l'innovation », a multiplié les investigations empiriques et a mis en évidence l'existence d'externalités technologiques locales. Ces résultats a priori solides ont largement contribué à faire émerger l'idée selon laquelle il convient d'être proche physiquement pour collaborer. Plus récemment cependant, différents auteurs se sont interrogés sur leur validité, les investigations empiriques à la base de la thèse du local ayant établi quasi-exclusivement leurs travaux à une échelle spatiale restreinte en considérant le local comme le lieu privilégié des collaborations pour l'innovation. Une nouvelle vague d'analyses empiriques a alors montré que si la dimension locale existe, elle est loin d'être exclusive (pour une synthèse, voir FERRU (2009)). 
Au niveau théorique, un certain nombre de travaux (RALLET et TORRE (2005) ; BOSCHMA (2005) ; BoschmA et FRENKEN (2009) ; BouBA-OLGA et GROSSETTI (2008)) expliquent cette diversité des échelles spatiales par le caractère pluriel de la proximité et montrent ainsi que " the proper impact of the geographical dimension must be more precisely assessed in relation to other types of proximity » (Autant-Bernard et al. (2007), p.343), telles que la proximité sociale, technologique, organisationnelle ou encore institutionnelle. Au niveau empirique, s'appuyant sur des bases de données véritablement relationnelles, les auteurs testent aujourd'hui le rôle de différents déterminants théoriques et l'effet respectif des proximités (GROSSETTI et NGUYEN, (2001); MAGGIONI et al. (2007); ABRAMOVSKY et SIMPSON (2008) ; FRENKEN et al. (2009) ; LAURSEN et al. (2010); HOEKMAN et al. (2010); FERRU (2010)).

L'objectif de cet article est de s'inscrire dans le prolongement de ces travaux, en les complétant sur différents points. D'un point de vue théorique, d'abord, la plupart des travaux pose la question de la dimension spatiale des collaborations en termes de choix de localisation. Or, dans tout un ensemble de cas, les acteurs de l'innovation s'inscrivent dans une logique de projet souvent multi-partenarial, ils ne cherchent pas à être localisés de façon permanente à coté de leur partenaire, mais plutôt à gérer leurs différentes collaborations en étant à la fois ici et ailleurs et en s'appuyant pour cela soit sur des dispositifs de coordination à distance, soit sur des proximités temporaires (RALLET et TORRE (2005)). La dimension spatiale des collaborations est dès lors moins un problème de choix de localisation qu'un problème de choix d'un collaborateur. Nous proposons donc dans une première partie de développer une telle modélisation, en nous inspirant des modèles d'urnes de Polya proposés par ARTHUR (1989, 1990) pour analyser les choix d'adoption des technologies ou des localisations. Le modèle proposé met en évidence le rôle potentiel de la géographie des ressources, d'une part, et de l'histoire des collaborations passées, d'autre part, des effets de lock-in géographiques (externalités technologiques locales) ou résiliaires (externalités technologiques non locales) pouvant se manifester.

D'un point de vue empirique, ensuite, les travaux recensés n'intègrent que rarement l'existence potentielle d'effets de structure, notamment d'effets régionaux ou sectoriels. De plus, ils ne peuvent se prononcer sur l'évolution de la dimension spatiale des collaborations, faute de données sur longue période. Nous proposons de combler partiellement ces lacunes en nous appuyant sur une base de données sur longue période (1981-2006) rassemblant l'ensemble des contrats Cifre signés entre des laboratoires publics et des entreprises, pour lesquels nous disposons notamment de la localisation géographique, du secteur d'appartenance (pour les entreprises) et du domaine scientifique (pour les laboratoires).

L'exploitation de cette base dans la deuxième partie de l'article montre d'abord l'inertie de la dimension spatiale des collaborations, la part des contrats intra-régionaux oscillant faiblement, sur toute la période, autour de $50 \%$. Nous testons ensuite un modèle probit afin d'expliquer le caractère local/non local des collaborations, qui permet de confirmer l'inertie des évolutions et de mettre en évidence l'existence d'effets géographiques marqués pour certaines régions françaises et, comparativement, la faiblesse des effets de secteur ou de domaine scientifique. Nous montrons enfin que ces effets régionaux s'expliquent pour partie par la géographie particulière des ressources : le nombre de contrats entre les régions est d'autant plus important que la tailler économique et scientifique des régions est elle-même importante. Les propositions principales de notre modélisation (importance de la géographie des ressources et de l'histoire des collaborations passées) paraissent donc validées.

\section{$-2-$}

\section{Un modèle d'adoption des collaborateurs}

Pour identifier les principaux déterminants des choix de collaboration pour l'innovation, nous nous inspirons des modèles d'urnes de Polya, utilisés pour l'adoption des technologies (ARTHUR (1989)) ou des localisations (ARTHUR (1990)). Nous focalisant ensuite sur les contrats Cifre reliant des entreprises et des laboratoires de recherche, nous considérons la localisation des acteurs comme donnée et irréversible : les contrats Cifre sont en effet signés 
pour une durée de 3 ans, ce sont les doctorants des laboratoires qui rejoignent les entreprises, les entités concernées ne modifiant pas leur localisation. Cette hypothèse ne vaut pas que pour ce type de collaboration : nombre des relations science-industrie, plus généralement nombre des collaborations pour l'innovation, obéissent de plus en plus à des logiques de projets, qui rendent le coût d'une co-localisation prohibitif. Le caractère multipartenarial des collaborations, les possibilités d'échange à distance, tout comme le recours fréquent à une proximité temporaire (RALLET et TORRE (2005)) conduisent également à poser le problème du choix du collaborateur comme différent d'un problème de choix de localisation.

Dans cette perspective, nous considérons une économie composée de $\mathrm{R}$ régions, dans lesquels sont localisés des entreprises $x$ et des laboratoires $y$. On note $x_{i r}$ l'entreprise $i=1$ à $X_{r}$ de la région $r$ et $y_{j r}$ le laboratoire $j=1$ à $Y_{r}$ de la région $r$. Le nombre total d'entreprises est $X=\sum_{r} X_{r}$, le nombre total de laboratoires est $Y=\sum_{r} Y_{r}$.

On considère qu'à chaque période $t=1$ à $T$, l'entreprise $x_{i r}$ noue un certain nombre de collaborations avec des laboratoires $y$, en fonction d'une propension à collaborer identique pour toutes les firmes, notée $p$. Le nombre total de collaborations initiées par les entreprises, pour chaque période $t$, sera donc, pour l'économie dans son ensemble, égal à $p X$ et, pour chaque région $r$, égal à $p X_{r}$.

\subsection{Le rôle de la géographie des ressources}

Soit $C\left(x_{i r 1}, y_{j r 2}\right)$ le nombre de collaborations entre la ième entreprise de la région $r 1$ et le jième laboratoire de la région $r 2$. On notera $C_{r 1, r 2}$ le nombre total de collaborations entre des entreprises de la région $r 1$ et des laboratoires de la région $r 2 . C_{r 1, r 1}$ est logiquement le nombre de collaborations intra-régionales.

Dans la configuration de base du modèle, nous supposons que la profitabilité de la collaboration est la même quels que soient l'entreprise et le laboratoire :

$$
\pi\left(x_{i r 1}, y_{j r 2}\right)=\underline{\pi} \forall i, j, r 1, r 2
$$

La probabilité qu'une entreprise $x_{i r 1}$ collabore avec un laboratoire $y_{j r 2}$ est alors la même quels que soient l'entreprise et le laboratoire. Le nombre de collaborations entre des entreprises de la région $r 1$ et des laboratoires de la région $r 2$ dépendra dès lors uniquement de la répartition spatiale des deux types d'acteurs. Plus précisément, compte tenu de l'ensemble des hypothèses posées, nous obtenons :

$$
C_{r 1, r 2}=p X_{r 1} \frac{Y_{r 2}}{Y}=A X_{r 1} Y_{r 2}
$$

Où $A$ est un coefficient de proportionnalité égal à $\frac{p}{Y}$

Symétriquement, le nombre de collaborations entre des entreprises de la région $r 2$ et des laboratoires de la région $r 1$ sera:

$$
C_{r 2, r 1}=p X_{r 2} \frac{Y_{r 1}}{Y}=A Y_{r 1} X_{r 2}
$$

Sur la base de ces éléments, nous pouvons calculer un degré de localisme des entreprises de la région $r 1$ égal à la part des collaborations intra-régionales dans l'ensemble des collaborations nouées par des entreprises de la région $r 1$, notée $c_{x, r 1}$ :

$$
c_{x, r 1}=\frac{C_{r 1, r 1}}{\sum_{r} C_{r 1, r}}=\frac{p X_{r r} \frac{Y_{r 1}}{Y}}{\sum_{r} p X_{r 1} \frac{Y_{r}}{Y}}=\frac{Y_{r 1}}{Y}
$$

Symétriquement, le degré de localisme des laboratoires de la région $r 1$ sera : 


$$
c_{y, r 1}=\frac{C_{r 1, r 1}}{\sum_{r} C_{r, r 1}}=\frac{p X_{r 1} \frac{Y_{r 1}}{Y}}{\sum_{r} p X_{r} \frac{Y_{r 1}}{Y}}=\frac{X_{r 1}}{X}
$$

La part des collaborations intra-régionales dépend donc potentiellement, sous les hypothèses mentionnées, de la géographie des ressources scientifiques et économiques: plus le nombre de laboratoires et d'entreprises implantés dans la région est important, plus le nombre de collaborations intra-régionales sera lui-même important.

\subsection{Le rôle de la proximité spatiale}

Concentrons nous sur les collaborations entre les entreprises d'une région $r 1$ et les laboratoires d'une région $r 2$. Nous avons vu que leur nombre est égal à :

$$
C_{r 1, r 2}=p X_{r 1} \frac{Y_{r 2}}{Y}=A X_{r 1} Y_{r 2}
$$

On peut supposer que le coût de la mise en relation entre les entreprises et les laboratoires considérés dépend positivement de la distance géographique entre les deux régions, notée $D_{r 1, r 2}$. Le nombre attendu de collaborations s'écrira alors :

$$
C_{r 1, r 2}=A \frac{X_{r 1} Y_{r 2}}{D_{r 1, r 2}}
$$

La relation obtenue correspond à un modèle gravitaire, que nous testerons dans la deuxième partie. La distance entre les deux régions peut être estimée de différentes façons : nombre minimal de frontières régionales à franchir pour aller d'une région à l'autre, distance kilométrique entre les deux capitales régionales, distance temps entre ces deux mêmes capitales, etc.

\subsection{L'introduction des rendements croissants d'adoption}

Supposons maintenant que la profitabilité d'une collaboration entre une entreprise et un laboratoire augmente avec le nombre de collaborations passées entre ces deux acteurs. En reprenant les notations introduites plus haut, nous écrirons :

$$
\pi_{t}\left(x_{i r 1}, y_{j r 2}\right)=\underline{\pi}+\alpha C_{t}\left(x_{i r 1}, y_{j r 2}\right)
$$

La relation (8) est une relation dynamique: $C_{t}\left(x_{i r 1}, y_{j r 2}\right)$ correspond au nombre de collaborations antérieures entre les deux acteurs, $\pi_{t}\left(x_{i r 1}, y_{j r 2}\right)$ renvoie à la profitabilité de la période courante, avec $\underline{\pi}$ la profitabilité intrinsèque de la collaboration et $\alpha$ un paramètre renseignant sur la nature des rendements d'adoption du partenaire.

Selon la nature des rendements (selon le signe et la valeur de $\alpha$ ), différentes situations peuvent apparaître :

- Si $\alpha=0$, les rendements résultant de l'adoption du même partenaire sont nuls, le bénéfice retiré de la collaboration reste le même au cours du temps, on retrouve le modèle initial.

- Si $\alpha<0$, les rendements d'adoption diminuent : il existe des deséconomies d'adoption d'un partenaire au fur et à mesure de la reconduction des collaborations. Les acteurs auront tendance à ne pas réactiver leurs anciennes collaborations.

- Enfin, si $\alpha>0$, les rendements d'adoption sont croissants: les bénéfices retirés de la collaboration avec le même partenaire augmente avec le nombre de collaborations établies au cours du temps. Les acteurs auront donc intérêt à reconduire leurs partenariats passés. Au-delà d'un certain nombre de collaborations, la probabilité que l'entreprise adopte toujours le même laboratoire tend ainsi vers 1 et symétriquement vers 0 pour les autres partenaires. 
L'existence de rendements croissants n'est pas systématique mais elle semble constituer une régularité importante dans la mise en relation des acteurs. Cette dernière hypothèse apparait en effet recevable dans tout un ensemble de cas, en raison d'économies d'adoption d'un partenaire ou d'externalités inter-organisationnelles. Les rendements croissants d'adoption trouvent deux sources essentielles entre lesquelles il est difficile de discriminer : 1) au fur et à mesure de leurs collaborations, l'entreprise et le laboratoire apprennent à travailler ensemble (learning) et à connaître précisément leur domaine de recherche respectif ; 2) lorsque les porteurs de projets s'en remettent à des anciens collaborateurs, le coût de la recherche du partenaire devient quasiment nul ; de même les coûts de contrôle et d'opportunisme anticipés sont fortement réduits car les partenaires évaluent le degré de confiance qu'ils peuvent accorder à l'autre.

La formulation retenue de la profitabilité de la collaboration permet de retrouver le modèle d'adoption des technologies d'ARTHUR (1989), la différence étant qu'il ne s'agit plus ici d'adopter une technologie, mais un collaborateur. En suivant les résultats de son modèle, on en déduit que chaque entreprise va être progressivement enfermée dans une relation bilatérale avec l'un des laboratoires (effet de lock-in), sans que l'on puisse prédire à l'avance avec lequel.

Cet enchaînement explicatif ne signifie pas que les entreprises auront un seul et unique partenaire scientifique et/ou que l'adoption d'un partenaire empêche parallèlement la formation de nouvelles collaborations. En effet, les entreprises peuvent avoir besoin de différentes ressources dans le cadre de leur projet ou de différents partenaires pour une même ressource.

Sans autre hypothèse, cet enfermement inter-organisationnel n'a pas d'implication sur la géographie des collaborations, c'est seulement si l'on suppose l'existence d'externalités entre acteurs que cette géographie sera affectée. Une hypothèse possible consiste par exemple à supposer que le développement de relations entre une entreprise de la région 1 et un laboratoire de la région 2 profite à l'ensemble des entreprises de la région 1. Le profit entre deux acteurs s'écrira alors :

$$
\pi\left(x_{i r 1}, y_{j r 2}\right)=\underline{\pi}+\alpha C_{t_{r 1, r 2}}
$$

Une hypothèse alternative consiste à supposer l'existence d'externalités non locales. Supposons par exemple qu'une partie des entreprises appartiennent à un même réseau, et que des externalités de connaissance se diffusent au sein, et uniquement au sein de ce réseau. L'enfermement collectif ne sera pas spatial, mais résiliaire. Ces externalités non locales peuvent également s'expliquer par le jeu de dispositifs institutionnels spécifiques dédiés aux relations entre certaines régions. Ce n'est plus la proximité spatiale qui compte, mais une autre forme de proximité, que l'on peut qualifier de socio-économique (BOuBAOLGA et GROSSETTI (2008)).

\section{$-3-$}

\section{Analyse empirique de la dimension spatiale des contrats Cifre}

Notre travail empirique s'appuie sur une base de données sur les contrats de recherche science-industrie dans le cadre d'une convention industrielle de formation par la recherche (Cifre) $)^{i}$. Les bourses Cifre apparaissent comme un indicateur pertinent pour l'analyse des collaborations pour l'innovation même si elles n'en capturent qu'une partie, puisqu'elles correspondent à un sous-ensemble des seules relations science-industrie.

La base rassemble l'ensemble des contrats Cifre établis sur la période 1981-2006 pour la France entière, soit au total 14669 contrats. Différentes informations relatives à ces contrats sont renseignées (cf. la présentation des données dans l'encadré suivant), notamment i) le secteur d'activité de l'établissement (décomposition en 18 secteurs), ii) le domaine scientifique du laboratoire (26 domaines), iii) la localisation du laboratoire (par commune, département et région), iv) la localisation de l'établissement (idem). Nous avons réduit 
l'analyse aux régions française de la métropole, hors Corse, soit 21 régions. Le nombre de contrats passe alors à 14135.

\section{Encadré : Les contrats Cifre}

Le dispositif CIFRE - Conventions Industrielles de Formation par la REcherche subventionne depuis 1981 toute entreprise de droit français qui emploie un doctorant pour le placer au cœur d'une collaboration de recherche avec un laboratoire public. Les CIFRE sont intégralement financées par le ministère de l'Enseignement supérieur et de la Recherche.

Les établissements qui contractent avec les laboratoires universitaires dans le cadre de CIFRE sont pour l'essentiel des établissements appartenant à des groupes (dans plus de $41 \%$ des cas) et des entreprises indépendantes de moins de 500 salariés (dans plus de $35 \%$ des cas). Ces établissements appartiennent à différents secteurs d'activité dont certains apparaissent dominants : les quatre premiers secteurs - le secteur du matériel électrique et électronique, les services, la parachimie et la construction mécanique - concentrent plus de $55 \%$ des contrats. Cette tendance est par ailleurs très stable. Du coté des laboratoires, on retrouve également quelques secteurs majeurs dans ces collaborations science-industrie: les laboratoires d'informatique, de physique, de SHS et de chimie rassemblent près de $40 \%$ des contrats CIFRE. On retrouve là-encore une très faible variation de ces différentes parts au cours du temps, hormis une légère baisse des principaux domaines scientifiques.

Bien que les établissements et les laboratoires de chacune des régions établissent des contrats CIFRE, on observe que ce sont notamment les acteurs des régions île-de-France, Rhône-Alpes, Midi-Pyrénées et PACA qui sont le plus actifs en termes de collaborations science-industrie puisqu'ils établissent plus de $65 \%$ de l'ensemble des contrats. Cette tendance est là-encore relativement stable au cours de la période d'étude. On observe par ailleurs que la concentration régionale des contrats est plus importante du coté des établissements que du coté des laboratoires: les établissements des quatre premières régions rassemblent $70 \%$ des contrats contre $62 \%$ pour les laboratoires universitaires.

Pour nous prononcer sur la dimension spatiale des collaborations, nous avons travaillé d'une part sur la part des relations intra-régionales et d'autre part sur la matrice des relations interrégionales. Dans un premier temps, nous nous focalisons sur l'évolution de la dimension spatiale des collaborations. Nous présentons ensuite un modèle probit, afin d'identifier d'éventuels effets structurels. Nous montrons enfin le rôle de la géographie des ressources pour la dimension spatiale des collaborations.

\subsection{L'inertie de la dimension spatiale des collaborations}

Pour nous prononcer sur la part des collaborations intra-régionales, nous avons construit une variable notée LOC, qui peut prendre deux valeurs : 1 si la relation est intra-régionale, 0 sinon. On peut alors calculer pour chaque région, secteur, domaine, etc., la part des contrats intra-régionaux dans l'ensemble des contrats et analyser son évolution.

Sur l'ensemble de la période et pour l'ensemble des régions, la part des contrats intrarégionaux est de $52,4 \%$. A l'échelle des départements, elle tombe à $29,1 \%$ et à $1,7 \%$ au niveau communal. La figure 1 reprend l'évolution de ces indicateurs, au niveau régional et départemental, ainsi que l'évolution du nombre de contrats Cifre.

Figure n9: Evolution du nombre de contrats et éche lle spatiale des collaborations 


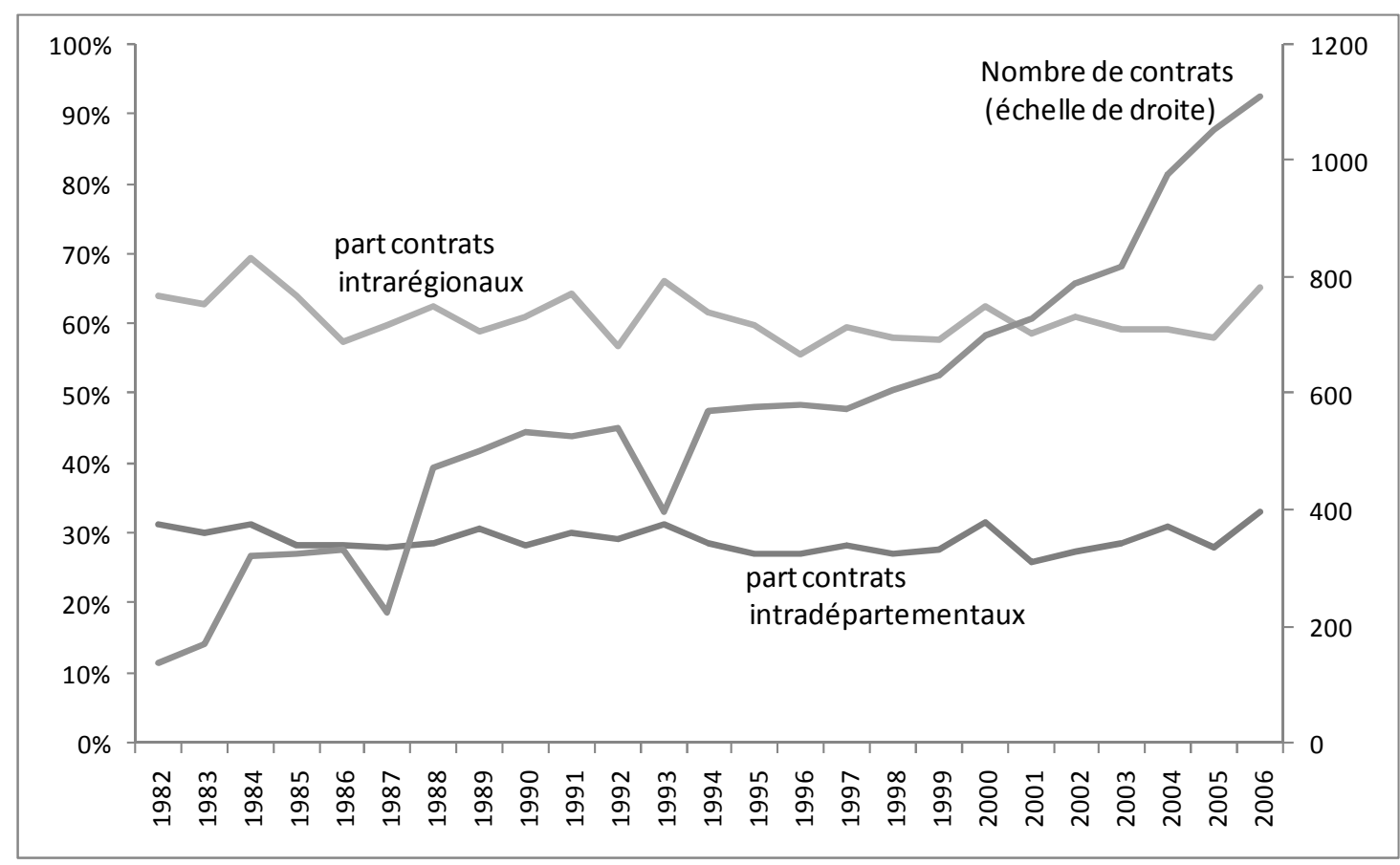

Premier résultat important: la proportion de contrats «locaux » à ces deux échelles est remarquablement stable, en dépit de l'évolution soutenue du nombre de contrats. Pour juger plus précisément de cette stabilité, nous avons testé économétriquement l'existence d'une tendance linéaire. L'équation à tester est :

$$
L O C_{r, t}=\alpha+\beta t+\mu_{t}
$$

Où $L O C_{r, t}$ est le taux de localisme à la date $t$ pour la région $r$ (ou un ensemble de régions), $t$ est le temps et $\mu_{t}$ est un terme d'erreur.

II s'avère que $\beta$ est non significatif pour la France entière, ce qui confirme la stabilité du localisme pour l'ensemble des régions sur la période d'étude. Autre résultat important, à un niveau géographique plus fin, on observe que le localisme, coté laboratoire, n'est pas stable ( $\beta$ est significatif au seuil de 1\%) pour la province et la région lle-de-France : on observe une tendance linéaire décroissante pour la première et inversement une tendance linéaire croissante pour la seconde. Ceci pourrait s'expliquer par l'évolution (défavorable) de la densité de la région lle de France relativement à la province, autrement dit par la géographie des ressources, ce que nous chercherons à vérifier dans le paragraphe suivant. Au contraire, coté établissement, $\beta$ n'est pas significatif, révélant la stabilité du localisme pour la province et l'lle-de-France.

Nous avons également effectué ce test pour chacune des régions : 11 sur 21 ne présentent pas de trend linéaire significatif côté établissement, et 14 sur 21 côté laboratoire. II convient toutefois de rester très prudent quant à ces résultats, les régions, hormis Rhône-Alpes, MidiPyrénées et l'lle de France, rassemblant un nombre de contrats par année relativement faible. Le tableau suivant résume ces différents résultats.

Tableau $n 9$ : Stabilité du localisme pour chacune des régions

\begin{tabular}{|c|c|c|}
\cline { 2 - 3 } \multicolumn{1}{c|}{} & Coefficient positif & Coefficient négatif \\
\hline \multirow{4}{*}{$\begin{array}{c}\text { Coté } \\
\text { établissement }\end{array}$} & $\begin{array}{c}\text { Auvergne, Bourgogne, Bretagne, } \\
\text { Haute-Normandie, Pays de la Loire, } \\
\text { PACA, Poitou-Charentes, Alsace, lle- } \\
\text { de-France*, Aquitaine**, Centre***, } \\
\text { Champagne-Ardenne*** }\end{array}$ & $\begin{array}{c}\text { Limousin, Languedoc-Roussillon, Basse- } \\
\text { Normandie, Midi-Pyrénées*, Franche- } \\
\text { Comté*, Lorraine**, Nord-Pas-de-Calais**, } \\
\text { Picardie***, Rhône-Alpes*** }\end{array}$ \\
\hline Coté & Midi-Pyrénées, Aquitaine, Auvergne, & Pays de la Loire, Limousin, Lorraine, Basse- \\
\hline
\end{tabular}




\begin{tabular}{|l|c|c|} 
laboratoire & $\begin{array}{c}\text { Bourgogne, Bretagne, Centre, } \\
\text { Champagne-Ardenne }\end{array}$ & $\begin{array}{c}\text { Normandie, Franche-Comté, Rhône-Alpes, } \\
\text { Haute-Normandie, Picardie*, Poitou- } \\
\text { Charentes*, Alsace**, Languedoc- } \\
\text { Roussillon***, Nord-Pas-de-Calais***, } \\
\text { PACA*** }\end{array}$ \\
\hline
\end{tabular}

Notes: ${ }^{*} P<0,1,{ }^{* *} P<0,05,{ }^{* * *} P<0,01$

Pour compléter sur cette question de l'inertie des évolutions, nous avons procédé à une analyse basée sur la matrice des relations interrégionales. A partir de cette matrice, nous pouvons calculer le profil régional des laboratoires (profils lignes) et des entreprises (profils colonnes), puis tester la stabilité des profils obtenus pour deux périodes différentes, 19811993 et 1994-2006. Pour cela, nous avons réalisé deux tests non paramétriques et plus précisément des tests de comparaison de distribution de Kolmogorov-Smirnov et de Wilcoxon. II s'avère que la distribution des collaborations est comparable entre les deux sous périodes. Autrement dit, pour chacune des régions, les collaborations inter-régionales ne connaissent pas d'évolution significative, ce qui confirme l'inertie de la dimension spatiale des collaborations. II n'est cependant pas possible, compte tenu des données disponibles, de se prononcer sur les déterminants d'une telle inertie : il peut s'agir d'une tendance à la reproduction des relations passées ou encore du jeu de la géographie des ressources. Nous montrons plus loin le rôle important de la géographie des ressources.

\subsection{Identification des effets de structure : un modèle probit}

Afin d'estimer l'impact des déterminants structurels sur la dimension spatiale des collaborations, nous avons testé un modèle probit avec $L O C_{k}^{*}$ la variable latente et $L O C_{k}$ la variable binaire associée, qui prend la valeur 1 si le $k^{\text {ième }}$ contrat est signé entre deux contractants de la même région et la valeur 0 sinon.

$$
L O C_{k}=\left\{\begin{array}{c}
1 \text { si } \operatorname{LOC}_{k}^{*} \geq 0 \\
0 \text { sinon }
\end{array}\right.
$$

Avec $L O C_{k}^{*}=\alpha+Z_{k} \beta+\mu_{k}$

$Z$ est la matrice des variables explicatives et $\beta$ le vecteur des paramètres associés.

Les variables explicatives sont le secteur d'appartenance de l'entreprise (SECTEUR), le domaine scientifique du laboratoire (DOMAINE), la localisation de l'entreprise (REG_ETAB) et la localisation du laboratoire (REG_LAB) et les dates de signature des contrats, classées en cinq périodes d'amplitude égale (PERIODE). Une dernière variable nous renseigne sur l'appartenance à un groupe (GROUPE). Pour les régions, aussi bien du coté des laboratoires que des établissements, la modalité de référence est la région Aquitaine. Pour les secteurs d'activité et les domaines scientifiques, les modalités de référence sont respectivement l'énergie et l'informatique. L'existence de données manquantes pour certains contrats réduit notre échantillon d'étude : le probit se fonde ainsi sur un échantillon de 10263 contrats.

Tableau n2: Résultats du modèle probit (Odd ratios significatifs)

\begin{tabular}{|c|c|c|}
\hline & Effets positifs & Effets négatifs \\
\hline \multicolumn{3}{|l|}{ Période } \\
\hline 1981-1985 & \multicolumn{2}{|c|}{ Modalité de référence } \\
\hline $1995-2000$ & & 1.35 \\
\hline 2000-2006 & & 1.26 \\
\hline Groupe & & 1.27 \\
\hline \multicolumn{3}{|c|}{ Localisation du laboratoire } \\
\hline Alsace & & 1.76 \\
\hline Aquitaine & \multicolumn{2}{|c|}{ Modalité de référence } \\
\hline
\end{tabular}


Auvergne

Basse-Normandie

Centre

île-De-France

7.69

Languedoc-Roussillon

Limousin

Lorraine

Poitou-Charentes

Localisation de l'établissement

Alsace

Aquitaine

1.72

Auvergne

Modalité de référence

Basse-Normandie

Bourgogne

Centre 4.36

Champagne-Ardenne

Franche-Comté

Haute-Normandie

île-De-France

6.46

Languedoc-Roussillon

2.88

Lorraine

3.41

Midi-Pyrénées

1.76

Picardie

Secteurs d'activité

Energie

Modalité de référence

Métallurgie

I.A.A.

Services

1.57

Domaine scientifique

Informatique

Modalité de référence

Instrumentation (Hors G.B.M.)

Génie Electrique

1.71

Métallurgie

1.68

Mécanique

Mécanique Des Fluides

Energétique

Chimie

G.B.M

S.H.S.

Biotechnologies

Seules les variables significatives au seuil de 5\% sont reprises dans ce tableau. Effets positifs : par rapport à l'Aquitaine, une localisation du laboratoire en lle de France multiplie par 7.69 la probabilité de collaborer au sein de la région. Effets négatifs: par rapport à l'Aquitaine, une localisation du laboratoire en Alsace divise par 1.76 la probabilité de collaborer localement.

Plusieurs résultats peuvent être soulignés : 
i) S'agissant de la dimension temporelle, les deux dernières périodes exercent une influence négative sur la probabilité de collaborer localement, comparativement à la première période, mais l'effet est assez modéré. Un test complémentaire d'égalité des coefficients des différentes modalités de la variable PERIODE confirme cette faible influence, puisque l'hypothèse d'égalité des coefficients ne peut être rejetée ( $p$-value $=0.1464$ ). Les périodes ont donc une influence très faible sur les collaborations locales, ce qui semble confirmer la relative stabilité de la géographie des collaborations observée précédemment et infirmer la tendance à l'agglomération spatiale observée par certains (GROSSETTI et NGUYEN (2001)).

ii) S'agissant des effets de spécialisation sectorielle, ils sont plus marqués côté domaines scientifiques (12 domaines sur 20 ont des coefficients significatifs) que côté industrie (6 secteurs sur 15). Le secteur industriel des services, d'une part, le domaine scientifique des sciences humaines et sociales, d'autre part, sont les seuls à jouer positivement sur la probabilité de collaborer localement. On ne retrouve pas de différences significatives entre les domaines relatifs aux sciences de la vie et aux sciences physique telles qu'observés par PONDS et al. (2007).

iii) L'appartenance à un groupe influe négativement sur la probabilité de collaborer localement. La valeur du odd ratio est cependant assez modérée. Cette influence peut sans doute résulter du fait que l'appartenance à un groupe ouvre des horizons de collaborations au-delà de la région d'appartenance de l'entreprise impliquée, celle-ci pouvant bénéficier au réseau de relations de l'ensemble des entités contrôlées par la société mère.

iv) Les effets régionaux sont marqués, côté laboratoire comme côté entreprise. Soulignons notamment le coefficient positif très significatif des laboratoires localisés en Ile-de-France, confirmant ainsi l'« effet capitale » observé par HOEKMAN et al. (2010) et GROSSETTI et NGUYEN (2001), ainsi que les valeurs négatives significatives pour les entreprises localisées dans des régions limitrophes de l'lle-de-France (Haute-Normandie, Champagne-Ardenne, Bourgogne, Picardie, Centre).

Ce dernier résultat nous semble particulièrement intéressant. Deux explications éventuellement complémentaires peuvent être avancées : soit les régions limitrophes à la région capitale bénéficient d'un effet de proximité vis-à-vis de l'lle-de-France, soit elles pâtissent d'un effet d'ombre. Pour vérifier la pertinence de ces explications, nous avons d'abord recherché si ce plus faible localisme des régions limitrophes pouvait s'expliquer par un nombre plus important de relations avec l'lle-de-France, le découpage administratif des régions masquant les effets de proximité géographique. Les données disponibles confirment cette hypothèse : alors que la moyenne des contrats avec l'lle-de-France est de $12 \%$ pour les régions de province, elle monte à $23 \%$ pour la Haute Normandie, $22 \%$ pour Champagne Ardennes et la région Centre, $19 \%$ pour la Picardie et $16 \%$ pour la Bourgogne ; seule l'Auvergne présente un taux supérieur $(25 \%)$ à ces régions. Le faible localisme de ces régions provient également d'un effet d'ombre (BROUILLAT et LUNG (2010)) en ne bénéficiant que d'un investissement modéré en recherche publique, ce qui renvoie à la question de la géographie des ressources et de l'existence d'effets-taille. Lorsqu'on mesure la dotation scientifique relative de ces régions (en rapportant leur poids dans le total des chercheurs à leur poids dans le PIB (données 2006)), on observe effectivement que la Champagne Ardenne, la Picardie et la Haute Normandie sont les trois régions les moins bien dotées, avec des indices respectivement de 32,33 et 38 , contre une moyenne pour les régions de province de 80.

\subsection{Le rôle de la géographie des ressources}

Afin de préciser l'interprétation des résultats observés dans le modèle probit précédent, nous proposons dans ce dernier point de nous pencher sur le rôle de la géographie des ressources. Dans le but de tester si le nombre de collaborations interrégionales et intrarégionales est lié à la taille scientifique et économique des régions, nous utilisons un modèle gravitaire $^{2}$, qui permet de relier le volume des collaborations interrégionales à la taille des 
régions et à la distance qui les sépare. II nous autorise ainsi à tester l'existence d'effets-taille et d'effets de distance (et symétriquement d'effets de proximité).

Nous proposons ici de tester la relation suivante, dérivée de la relation présentée dans la partie théorique :

$$
\ln C_{r 1, r 2}=\ln A+\alpha_{1} \ln \left(\frac{X_{r 1}}{X}\right)+\alpha_{2} \ln \left(\frac{Y_{r 2}}{Y}\right)-\beta \ln D_{r 1, r 2}+\gamma \ln I N T R A+\mu
$$

Méthodologiquement, compte tenu des caractéristiques des données mobilisées (données de comptage pouvant être considérées comme continues, nombre important de 0 dans la variable expliquée, transformation logarithmique), nous proposons d'estimer un tobit censuré simple.

Les variables explicatives de notre modèle sont les suivantes :

1) La distance spatiale, notée $D_{r 1, r 2}$, qui correspond à la distance géographique $r_{1}$ et $r_{2}$. Différents indicateurs peuvent être utilisés pour mesurer une telle distance. Trois spécifications ont été retenues : une matrice des contigüités, d'abord (nombre de frontières traversées pour se rendre d'une région à l'autre), le temps de transport par train entre les deux capitales régionales, ensuite, et la fréquence des trains (nombre de train par jour en moyenne), enfin.

Nous intégrons une dummy INTRA, qui ne figure pas dans le modèle théorique, mais qui permet de rendre compte de la spécificité des collaborations intra-régionales non prise en compte par la distance spatiale (qui est égale à 0 quand $r_{1}=r_{2}$ ), pour capturer les effets de co-localisation des partenaires. Elle est égale à 1 quand $r_{1}=r_{2}$ et égale à 0 quand $r_{1} \neq r_{2}$.

2) Les effets taille, qui renvoient à l'inégale dotation en ressources économique et scientifique des régions. Nous intégrons deux variables explicatives relatives à la taille des régions, $\ln X_{r 1}$ et $\ln Y_{r 2}$. La variable $\ln X_{r 1}$ est la taille économique relative de la région $r_{1}$. Pour mesurer la taille économique des régions, nous avons retenu le PIB et le nombre d'établissements. La variable $\ln Y_{r 2}$ est la taille scientifique relative de la région $r_{2}$. Elle est mesurée par le pourcentage de chercheurs publics par région. Etant donné que pour cette dernière variable seule des données sur la période 1997-2006 étaient disponibles, nous avons testé le modèle gravitaire sur ce seul intervalle.

Nous avons testé successivement deux modèles gravitaires. Un premier modèle se focalise exclusivement sur l'effet de la géographie des ressources. De ce fait, nous considérons que l'espace est neutre (la distance entre les régions est nulle). La taille économique est mesurée par trois indicateurs différents donnant lieu aux trois versions du modèle :

(1) Dans une première version du modèle, la taille économique des régions est évaluée par le nombre de chercheurs privés (données MENESR).

(2) Dans une deuxième version par le nombre d'établissements (données Unistatis).

(3) Dans une troisième version par le PIB (données INSEE), en moyenne sur la période d'étude.

Dans un second modèle nous intégrons le rôle de la distance, à deux niveaux : en intégrant, d'une part, la dummy INTRA pour capturer l'effet de la co-localisation des acteurs au sein de la même région, et en introduisant, d'autre part, une mesure de la distance interrégionale. Nous mesurons cette dernière de trois manières différentes, ce qui nous conduit à tester trois versions différentes de ce deuxième modèle.

(1) Nous testons dans une première version de ce second modèle le rôle de la distance géographique à partir d'une matrice des contiguïtés. Nous nous appuyons pour cela sur le 
nombre de frontières administratives entre chacune des régions, l'existence de frontières pouvant s'avérer déterminantes.

(2) Dans une seconde version, la distance est évaluée en termes de temps: nous nous utilisons le temps de transport par train nécessaire au déplacement entre chacune des capitales régionales.

(3) Dans la troisième et dernière version, nous intégrons à la précédente mesure de la distance, la fréquence des trains, qui accroit l'accessibilité entre les territoires. Dans cette dernière version, la distance entre deux régions est mesurée par le temps de transport par train entre les deux capitales régionales, divisé par le nombre quotidien de trains.

La dummy INTRA est présente dans les trois versions de ce second modèle.

Tableau n 3 : Résultats du modèle gravitaire pour le s collaborations interrégionales

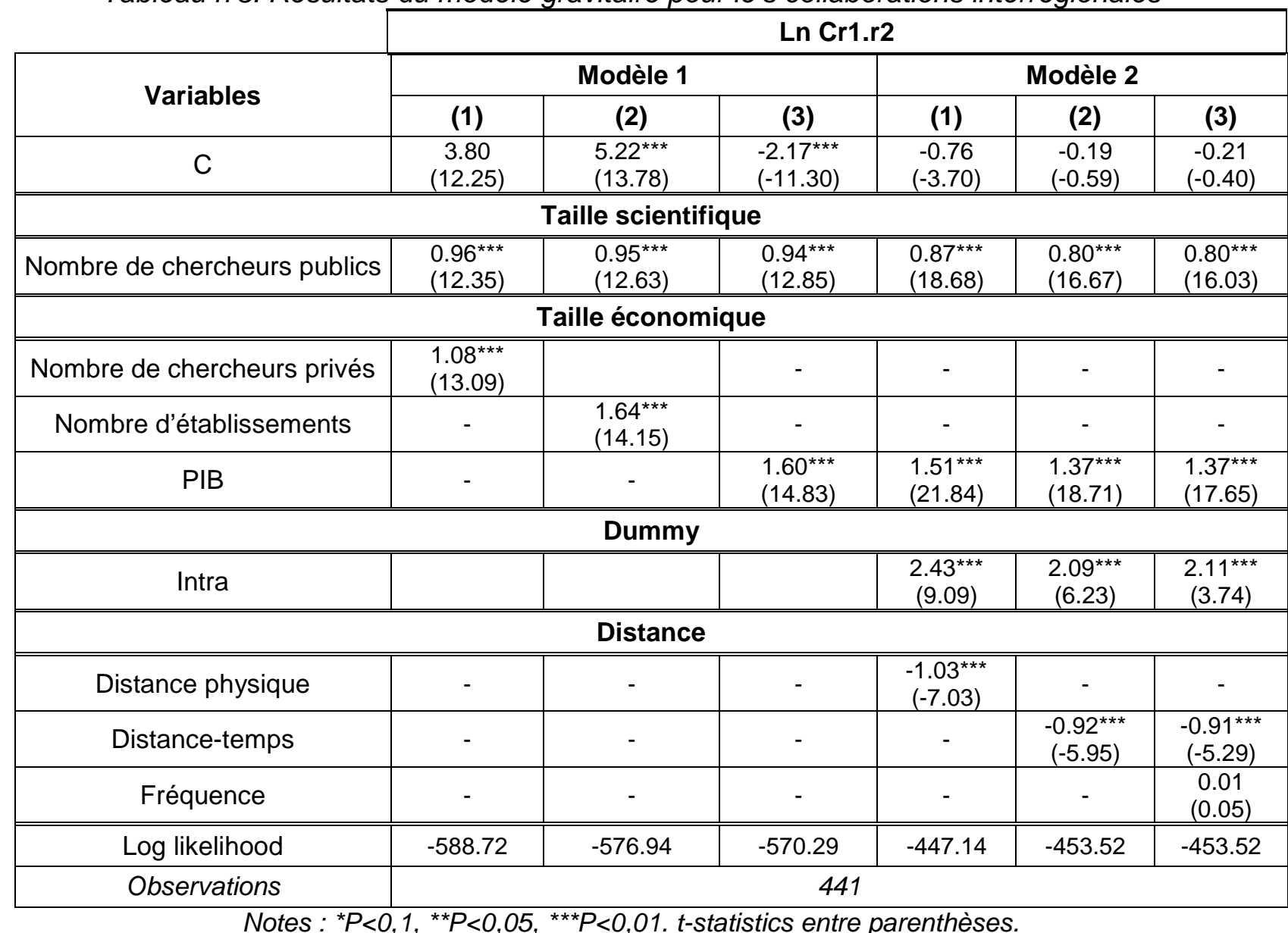

De façon générale, il apparaît que les modèles testés expliquent de manière satisfaisante le nombre de contrats entre les régions françaises, comme en témoigne le log de vraisemblance des différentes versions du modèle.

Le premier modèle rend compte du rôle positif et important de la géographie des ressources: la taille économique et scientifique des régions influence positivement le nombre de collaborations entre deux régions. Autrement dit, plus la taille des régions s'accroît et plus le nombre de collaborations augmente. Par ailleurs, le modèle révèle que la taille économique des régions a une influence plus grande dans la distribution spatiale des collaborations, relativement à la taille scientifique des régions : une hausse de $1 \%$ de la taille scientifique des régions entraine une augmentation de $0,95 \%$ environ du nombre de collaborations entre ces régions contre $1,6 \%$ pour la taille économique des régions. II 
convient de préciser toutefois que ce dernier résultat relatif à la taille économique des régions n'est valable qu'au regard du PIB ou du nombre d'établissements ; lorsque la taille économique est mesurée en nombre de chercheurs privés, l'élasticité est plus faible.

Dans le second modèle, la taille économique est mesurée uniquement par le poids du PIB national moyen sur la période 1997-2006. L'intégration de la distance entre les régions permet d'enrichir sensiblement l'explication de la géographie des collaborations. La variable muette INTRA, indicatrice des effets de co-localisation, est significative au seuil de $1 \%$, son élasticité est relativement importante, variant de 2,09 à 2,43 selon les versions du modèle. S'agissant de la distance interrégionale, son effet est également significatif quelle que soit sa mesure. Comme attendu, la distance joue négativement. En d'autres termes, une augmentation de $1 \%$ de la distance entre deux régions entraine une baisse d'environ $1 \%$ du nombre de contrats entre ces régions. Que la distance soit mesurée par le nombre de frontières ou par le temps de transport, l'effet reste le même. La prise en compte de la fréquence des trains n'apporte pas non plus d'éléments explicatifs supplémentaires, son coefficient n'étant pas significatif.

Au final, on observe que les effets-taille et les effets de distance structurent significativement la répartition spatiale des collaborations science-industrie. Ce modèle nous permet de confirmer nos hypothèses: les collaborations entre deux régions sont d'autant plus importantes que ces dernières sont denses en ressources scientifiques et économiques ; au contraire, plus les régions sont éloignées physiquement et moins le nombre de contrats entre ces deux régions est élevé.

\section{$-4-$ \\ Conclusion}

L'objectif de cet article était de compléter les travaux empiriques portant sur la dimension spatiale des collaborations pour l'innovation, en nous appuyant sur une base de données originale, portant sur une période relativement longue, et permettant de mesurer d'éventuels effets de structure.

Nous avons montré dans une première partie, sur la base d'un modèle d'urnes de POLYA, le rôle potentiel de la géographie des ressources, d'une part, et de l'histoire des collaborations passées, d'autre part. Les investigations empiriques menées ont permis de valider certaines des propositions avancées: i) on observe une forte inertie de la dimension spatiale des collaborations tout au long de la période d'étude, ii) on note également l'existence d'effets géographiques spécifiques, notamment pour l'lle de France et pour les régions limitrophes de la région capitale, iii) ces effets géographiques spécifiques s'expliquent pour partie par la géographie des ressources.

II conviendrait de compléter ces premières investigations, afin notamment de mieux comprendre l'inertie de la dimension spatiale des collaborations: au-delà du rôle de la géographie des ressources, celle-ci peut en effet s'expliquer par une tendance des acteurs à reconduire leurs collaborations passées. Des enquêtes menées auprès des acteurs impliqués dans les collaborations pour l'innovation seraient nécessaires pour mieux comprendre l'importance de ces effets d'inertie mais également les facteurs à l'origine de cette dépendance au sentier et ceux qui au contraire conduisent à une " bifurcation » dans la trajectoire des collaborations.

\section{Bibliographie}

ABRAMOVSKY L. et SIMPSON H., 2008, Geographical proximity and firm-university innovation linkages: evidence from Great Britain, Working paper CMPO.

ACS J., AUDRETSCH D. et FELDMAN M., 1991, "Real effects of academic research : comment », The American Economic Review, Vol. 82, n¹, pp. 363-367. 
ANSELIN L., VARGA A. et ACS Z., 1997, « Local geographic spillovers between university research and technology innovation ", Journal of Urban Economics, Vol. 42, issue 3, p. 422-448.

ARTHUR B., 1989, "Competing technologies, increasing returns and lock-in by small historical events », Economical Journal, Mars.

ARTHUR B., 1990, "When do increasing returns imply monopoly », Mathematical social sciences, Vol. 19, p.235-251.

AUDRETSCH D. et FELDMAN M., 1996. "Knowledge Spillovers and the Geography of Innovation and Production ", American Economic Review, Vol. 86, issue 3, p.630-640.

AUTANT-BERNARD C., MAIRESSE J. et MASSARD N., 2007, "Spatial knowledge diffusion through collaborative networks", Introduction to the special issue of Papers in Regional Science, Vol. 86 (3).

BERGMAN E., 2010, "Knowledge links between European universities and firms: A review », Papers in Regional Science, Vol. 89 (2), pp. 311-333.

BOSCHMA R. et FRENKEN K., 2009, «The spatial evolution of innovation networks. A proximity perspective ", In R. Boschma and R. Martin (eds) Handbook of Evolutionary Economic Geography, Cheltenham.

BOSCHMA R., 2005, "Proximity and innovation: a critical assessment », Regional studies, Vol. 39(1), pp.61-74.

BOUBA-OLGA O. et GROSSETTI M., 2008, “Socio-économie de proximité », Revue d’Economie Régionale et Urbaine, n\%3, p. 311-328.

BROUILLAT E. et LUNG Y., 2010, «Spatial distribution of innovative activities and economic performances : A geographical-friendly model », Working Papers of GREThA 2010-11.

CARAYOL N., 2003, "Objectives, agreements and matching in science-industry collaborations: Reassembling the pieces of the puzzle ", Research Policy, Vol. 32, pp. 887-908.

ETZKOWITZ H., WEBSTER A. et HEALEY P., 1998, "Capitalizing knowledge. New intersections of industry and academia », SUNY.

FELDMAN M.P., 1994. "The Geography of Innovation », Kluwer Academic Publishers, Dordrecht.

FELDMAN MP. et FLORIDA R., 1994, "The Geographic Sources of Innovation: Technological Infrastructure and Product Innovation In the United States », Annals of the Association of American Geographers, Vol. 84, pp. 210-229.

FERRU M., 2010, «Formation process and geography of science-industry partnerships : the case of the University of Poitiers ", Industry and Innovation, Vol. 17 (5).

FERRU M., 2009, "La géographie des collaborations pour l'innovation", Thèse ès sciences économiques, Université de Poitiers.

FRENKEN, K., HARDEMAN, S. et HOEKMAN, J., 2009, "Spatial scientometrics: towards a cumulative research program", Journal of Informetrics, Vol. 3 (3), pp. 222-232.

GIBBONS M., LIMOGES C., NOWOTNY H., SCHWARTZMAN S., SCOTT P. et TROW M., 1994, The new production of knowledge, The dynamics of science and research In contemporary societies, Sage, Londres

GODIN B. et GINGRAS Y., 1999, "L'impact de la recherche en partenariat sur la production scientifique ", Dossier de recherche de l'AUCC, Vol. 3 (3). 
GROSSETTI M. et NGUYEN D., 2001, « La structure spatiale des relations science-industrie en France: l'exemple des contrats entre les entreprises et les laboratoires CNRS ", Revue d'Economie Régionale et Urbaine, Vol. 2, pp. 311-328.

HAGEDOORN J. et ROIJAKKERS N., 2006, «Inter-firm R\&D partnering in pharmaceutical biotechnology since 1975: trends, patterns, and networks », Research Policy, vol. 35(3), pp. $431-446$

HOEKMAN J., FRENKEN K. et TIJSSEN R., 2010, "Research collaboration at a distance : changing spatial patterns of scientific collaboration within Europe ", Research Policy, Vol. 39 (5), pp. 662-673

JAFFE A., 1989, «Real effects of academic research », The American Economic Review, Vol. 79(5), p. 957-970.

JOLY P.B. et MANGEMATIN V., 1996, Profile of public laboratories, industrial partnerships and organisation of R\&D, Research Policy, 25, 901-922

LALLEMENT R., MOUHOUD EM. et PAILLARD S., 2007, «Polarisation et internationalisation des activités d'innovation: incidences sur la spécialisation technologique des nations ", In A. Rallet et A. Torre A. (dir), Quelle proximité pour innover?, Ed. L'Harmattan.

LAURSEN, K., REICHSTEIN, T. et SALTER A., 2010, « Exploring the effect of geographical proximity and university quality on university-industry collaboration in the UK», Regional Studies, pp. 1-17.

LUNG Y., 1997, "Organisation spatiale et coordination des activités d'innovation des entreprises », Rapport pour le Commissariat Général du Plan, octobre.

MAGGIONI M., NOSVELLI M. et UBERTI T., 2007, "Space versus networks in the geography of innovation », Papers in Regional Science, vol. 86(3), pp. 471-493,

MAGGIONI M. et UBERTI T., 2007, «Inter-regional knowledge flows in Europe: an econometric analysis », In Frenken K. (eds) Applied evolutionary, economics and economic geography, Edaward Elgar, Cheltenhal, UK.

PONDS R., VAN OORT F. et FRENKEN K., 2007, "The geographical and institutional proximity of research collaboration », Regional Science, Vol. 86 (3), pp. 423-443.

PUGA D., 1999, «The rise and fall of regional inequalities », European Economic Review, Vol. 43 (2), pp. 303-334.

RALLET A. et TORRE A., 2005., "Proximity and localization », Regional studies, Vol. 39(1), p.47-59.

SAXENIAN A., 1994, Regional advantage: culture and competition In Silicon Valley and Route 128, Cambridge, Harvard University Press.

SESSI, 2006. «Un quart des entreprises innovent. Souvent moyennes ou grandes, elles réalisent $60 \%$ du chiffre d'affaire », 4 pages, Aout.

WAGNER C. et LEYDESDORFF L., 2005. "Network structure, self-organization, and the growth of international collaboration in science", Research Policy, Vol. 34(10), pp. 16081618. 


\section{Notes}

1 - Cette base nous a été fournie par l'Association Nationale de la Recherche et de la Technologie, organisme qui gère ce type de convention au niveau national. Nous remercions Nadine Massard pour son intermédiation.

2 - Les modèles gravitaires sont utilisés généralement dans le cadre de l'analyse du commerce international ; ils ont été repris depuis quelques années pour apporter des éléments explicatifs à la géographie des collaborations (cf. Ponds et al. (2007) ; Maggioni et Uberti (2007) notamment). 